\title{
THE COLLINEATION GROUPS OF FREE PLANES
}

\author{
BY \\ REUBEN SANDLER
}

Free projective planes $\pi^{n}$ (generated by $n$ points on a line and two points off that line) have been studied for some time. Until now, however, virtually nothing was known about the collineation groups of the planes $\pi^{n}$ except that the groups possessed no central collineations. In this paper, the collineation groups will be studied. We shall show that the collineation group of $\pi^{2}$ has three generators and exhibit them. Then, the orbit under this group of those points which lie on generating quadrilaterals will be determined. Finally, a subgroup $H_{n}$ of the collineation group of $\pi^{n}(n>2)$ will be defined and it will be shown that there exists an integer $m$ such that for every $n>2, H_{n}$ is generated by at most $m$ collineations. It is the author's conjecture that $H_{n}$ is in fact the full collineation group of $\pi^{n}$, but the methods of the present paper do not seem to generalize to give this result.

1. Preliminaries. A set of points and lines and an incidence relation is said to form a projective plane if the following three axioms are satisfied:

I. Any two distinct points are incident with exactly one line.

II. Given any two distinct lines, there is exactly one point incident with both.

III. There exist four points, no three incident with one line.

If, on the other hand, the following two axioms are satisfied, the set is said to constitute a partial plane:

1. There exists at most one line through any two distinct points.

2. There is at most one point incident with any two distinct lines.

Given any partial plane $\pi_{0}$ which is not complete (projective), we can define new partial planes as follows: For any two points in $\pi_{0}$ not already connected by a line in $\pi_{0}$, we adjoin a new line. Let $L_{0}$ be the set of all new lines adjoined in this manner. Then, for any two lines in $\pi_{0} \cup L_{0}$ not already intersecting in a point of $\pi_{0}$, we adjoin a new point as their point of intersection. Let $\pi_{1}$ be the union of $\pi_{0}, L_{0}$, and the new points just adjoined. Clearly, $\pi_{1}$ is a partial plane. One can define $\pi_{2}, \pi_{3}, \cdots$ in an analogous manner, and set

$$
\pi=\bigcup_{i=0}^{\infty} \pi_{i},
$$

with the obvious incidences. Then $\pi$ is easily seen to be a complete plane. Any extension of a partial plane $\pi_{0}$ formed by letting each new point be the intersection

Presented to the Society, January 17, 1962; received by the editors April 10, 1962. 
of exactly two old lines, and each new line contain only two old points, is called a free extension of $\pi_{0}$.

We next state some lemmas which can be found in Hall [1, §4].

Lemma 1. The free extension of a partial plane to a complete plane is unique.

LEMMA 2. If $\pi_{2}$ is a free extension of $\pi_{1}$ and $\pi_{1}$ is a free extension of $\pi_{0}$, then $\pi_{2}$ is a free extension of $\pi_{0}$. The free extension of $\pi_{1}$ to a complete plane is the free extension of $\pi_{0}$ to a complete plane.

Definition. If $\pi_{1}, \pi_{2}$ are two partial planes, and if $\pi_{2} \subset \pi_{1}, \pi_{2}$ is said to be complete in $\pi_{1}$ if

(1) any line of $\pi_{1}$ containing two points of $\pi_{2}$ already is in $\pi_{2}$;

(2) any point of $\pi_{1}$ contained in two lines of $\pi_{2}$ already is in $\pi_{2}$.

LEMMA 3. If $\pi_{1} \supset \pi_{2}$ and $\pi_{2}$ is complete in $\pi_{1}$, then the free extension $\pi_{1}^{\prime}$ of $\pi_{1}$ contains as a subplane the free extension $\pi_{2}^{\prime}$ of $\pi_{2}$. If $\pi_{2} \neq \pi_{1}$, then $\pi_{2}^{\prime} \neq \pi_{1}^{\prime}$.

The free plane $\pi^{n}(n \geqq 2)$ is defined to be the free extension of the partial plane $\pi_{0}^{n}$ consisting of $n$ points on a line and two points not on that line to a complete plane.

Lemma 4. A $\pi^{n}$ contains $a \pi^{n+1}$ as a subplane.

LEMMA 5. If $n \neq m, \pi^{n}$ and $\pi^{m}$ are not isomorphic.

A collineation $\phi$ of a projective plane $\pi$ is a 1-1 point-to-point and line-to-line mapping of the plane onto itself which preserves incidences. In what follows we shall study the collineation groups of the free planes $\pi^{n}$.

2. General results. We begin this section by proving some rather elementary results which will allow us to get some insight into the structure of the collineation group of $\pi^{n}$. Let $A_{1}, A_{2}, \cdots, A_{n}, B_{1}, B_{2}$ be the points (of $\pi_{0}^{n}$ ) generating $\pi^{n}$, where the $A_{i}$ all lie on one line, and no other incidences hold.

THEOREM 1. Let $\phi$ be a collineation of $\pi^{n}$. Then $\phi$ is uniquely determined by its action on the points $A_{i} ; i=1,2, \cdots, n$, and $B_{j} ; j=1,2$.

Proof. This result can be proved by induction on the partial plane $\pi_{k}^{n}$, a free extension of $\pi_{0}^{n}$. For, if $L \in \pi_{k}^{n}, L \notin \pi_{k-1}^{n}$, then $L$ can be described as the unique line joining two points in $\pi_{k-1}^{n}$. If $\phi$ is already defined (by induction) on these two points, then $(L) \phi$ must be the unique line joining the images of the points. Similarly, the definition of $\phi$ is extended to points in $\pi_{k}^{n}$ but not in $\pi_{k-1}^{n}$.

The next question to which we address ourselves concerns the possible images of $\pi_{0}^{n}$ under a collineation, $\phi$, of $\pi^{n}$. The following theorem is evident. 
THEOREM 2. The image of $\pi_{0}^{n}$ under a collineation $\phi$ must be a configuration $C$ of $n$ points on one line and two points off that line. Furthermore, the subplane of $\pi^{n}$ generated by $C$ must be all of $\pi^{n}$.

Proof. The first statement follows from the fact that $\phi$ must preserve incidences, and the second, from the fact that $\phi$ must be onto.

A final theorem is needed before we can begin to characterize better the collineation group of $\pi^{n}$. If $\pi$ is a finite partial plane, let $P$ be the number of points of $\pi, L$ the number of lines, and $I$ the number of incidences defined by the points and lines of $\pi$. Hall has shown [1] that the number $2(P+L)-I$ is invariant under free extension. We call the number $\gamma(\pi)=2(P+L)-I$ the rank of $\pi$ and observe that the rank of a generating configuration of a $\pi^{n}$ can be computed as $\gamma\left(\pi_{0}^{n}\right)=n+6$.

THEOREM 3. Let $\pi^{n}$ be a free plane (of rank $\left.n+6\right)$. Let $\sigma_{0}$ be a finite subpartial plane of $\pi^{n}$ which is of rank $n+6$ and which generates all of $\pi^{n}$. Then $\sigma_{0}$ generates $\pi^{n}$ freely.

Proof. To $\sigma_{0}$, adjoin the lines which can be added and then the points. Call the union of $\sigma_{0}$, the new points and the new lines $\sigma_{1}$. Then $\sigma_{i}$ is defined inductively. If a new point (line) is always added as the intersection of two lines (points), the extension of $\sigma_{0}$ to $\pi^{n}$ will be free. If not, then in some $\sigma_{k}$, an intersection two lines (points) of $\sigma_{k-1}$ will be defined which does not create a new point (line). Thus an incidence is created, with no element being created, and we have $\gamma\left(\sigma_{k}\right)<\gamma\left(\sigma_{0}\right)=n+6$. Now for some $m, \sigma_{k} \subset \pi_{m}^{n}$. But if we extend $\sigma_{k}$ to $\pi_{m}^{n}$ by building outward from $\pi_{0}^{n}$ and adding those elements not in $\sigma_{k}$, the rank can never be increased since a new line (point) is always created as the intersection of at least two points (lines). Thus we would have $\gamma\left(\pi_{m}^{n}\right) \leqq \gamma\left(\sigma_{k}\right)<n+6$. But by Theorem 4.10 of Hall [1] we know that $\gamma\left(\pi_{m}^{n}\right)=n+6$, which implies that $\sigma_{0}$ must generate $\pi^{n}$ freely as asserted.

Because of Theorems 1,2 and 3, we can study in detail the collineation group of $\pi^{n}$ if we can determine all configurations of $n$ points on a line and two points off the line which generate all of $\pi^{n}$. For let $\left\{C_{i}\right\}$ be the set of all such configurations $\left(C_{1}=\pi_{0}^{n}\right)$. Then we have

THEOREM 4. If $n>2$, there is a group of collineations of $\pi^{n}$ isomorphic with $S_{n} \times S_{2}$, and if $n=2$, the corresponding group is isomorphic with $S_{4}$. Call this group $S^{n}$. Then if $G_{n}$ is the collineation group of $\pi^{n}$, we have

$$
G_{n}=\sum_{i} S^{n} T_{i}
$$

where $T_{i}$ is a collineation of $\pi^{n}$ which maps $\pi_{0}^{n}$ onto the configuration $C_{i}$.

Proof. If $n>2, S^{n}$ consists of those collineations generated by permuting the $n$ points $A_{i}$ among themselves in all possible ( $n$ !) ways, and by permuting $B_{1}$ and 
$B_{2}$ or leaving them fixed; $S^{2}$ is generated by the (4!) permutations of the points $A_{1}, A_{2}, B_{1}, B_{2}$ among themselves. Clearly, $S^{n}$ consists of all collineations of $\pi^{n}$ mapping $\pi_{0}^{n}$ onto itself. Let $\phi_{1}, \phi_{2}$ be two elements in the same left coset of $S^{n}$ in $G_{n}$. Then $\phi_{1}=\theta \phi_{2}, \theta \in S^{n}$. But this implies that $\phi_{1}$ and $\phi_{2}$ both map $\pi_{0}^{n}$ onto the same configuration $C_{i}$. Thus, $T_{i}$ is a coset representative of $S^{n}$ in $G_{n}$.

To help determine the desired configurations, we have

THEOREM 5. Let $C$ be any configuration of $n$ points on a line and two points off the line contained in a free extension of $\pi_{0}^{n}, \pi$. Then $C$ generates $\pi^{n}$ if and only if the minimal complete sub-partial plane of $\pi$ generated by $C$ is $\pi$ itself.

Proof. To $C$ adjoin (within $\pi$ ) points and lines until it is not possible to add any more elements. The set of points and lines so generated, $\pi^{\prime}$, will be a subpartial plane of $\pi$ which is complete in $\pi$, by the definition of $\pi^{\prime}$. By Lemma 3, if $C$ is to generate $\pi^{n}$, we must have $\pi^{\prime}=\pi$.

In concluding this section, some concepts will be introduced which will be of much help in what follows. If $\pi_{0}$ is a partial plane, let $L_{0}$ be the set of all lines which can be added to $\pi_{0}$ as in $\S 1$. Then if $P_{0}$ is the set of all new points which can be added to $\pi_{0}$ as the intersection of lines (two at a time) of $\pi_{0} \cup L_{0}$ which do not intersect in $\pi_{0}$, we shall call $P_{0}$ the set of diagonal points of $\pi_{0}$. Then, $\pi_{1}=\pi_{0} \cup L_{0} \cup P_{0}$. If $\pi_{0}=\pi_{0}^{n}$, the generating configuration for a free plane, we shall call the group $S^{n}$ the collineations of rank 0 , and define as the collineations of rank 1 that set of collineations which map $\pi_{0}^{n}$ into $\pi_{1}^{n}$. With these concepts, and Theorems 1,2 , and 3 at our disposal, we can proceed to an examination of the collineation groups of the planes $\pi^{n}$.

3. The case $n=2$. If $A_{1}, A_{2}, B_{1}, B_{2}$ are the four points of $\pi_{0}^{2}$, we let $L_{1}$ be the line containing $A_{1}$ and $A_{2}\left(L_{1}=A_{1} \cdot A_{2}\right), L_{2}=B_{1} \cdot B_{2}, L_{3}=A_{1} \cdot B_{1}, L_{4}=A_{2} \cdot B_{2}$, $L_{5}=A_{1} \cdot B_{2}, L_{6}=A_{2} \cdot B_{1}$. The configuration $\pi_{0}^{2}$ has three diagonal points: $a_{1}=L_{1} \cap L_{2}, a_{2}=L_{3} \cap L_{4}, a_{3}=L_{5} \cap L_{6}$. By inspection, one can check that there are exactly seven configurations of four points in $\pi_{1}^{2}$ which generate $\pi^{2}$. They are:

$$
\begin{aligned}
& C_{1}=\pi_{0}^{2}, C_{2}=\left\{a_{1}, a_{2}, A_{1}, B_{2}\right\}, C_{3}=\left\{a_{1}, a_{2}, B_{1}, A_{2}\right\}, \\
& C_{4}=\left\{a_{1}, a_{3}, A_{1}, B_{1}\right\}, C_{5}=\left\{a_{1}, a_{3}, A_{2}, B_{2}\right\}, C_{6}=\left\{a_{2}, a_{3}, A_{1}, A_{2}\right\}, \\
& C_{7}=\left\{a_{2}, a_{3}, B_{1}, B_{2}\right\}(1) .
\end{aligned}
$$

The investigations in this section will show that $G_{2}$ is generated by $S^{2}$ and any $T_{i}, i=2,3, \cdots, 7$.

Before proving our main results, one final concept is needed. If $C$ is a configuration of four points generating $\pi^{2}$, the ordered set of configurations

(1) The remaining sets of four points either have three points on a line, or generate proper subplanes of $\pi^{2}$. 
$\pi_{0}^{2}=C_{i_{1}}, C_{i_{2}}, \cdots, C_{i_{k}}=C$ is called a chain of configurations between $\pi_{0}^{2}$ and $C$ of length $k$ if (1) every $C_{i}$ is a configuration of four points generating $\pi^{2}$; and (2) $C_{i j+1}$ is contained in the seven points consisting of $C_{i j}$ and the diagonal points of $C_{i j}$. We can now state and prove

THEOREM 6. Let $C$ be a configuration of four points of $\pi^{2}$ generating $\pi^{2}$. If $C \subset \pi_{k}^{2}$, then there exists a chain of length $k$ or less between $\pi_{0}^{2}$ and $C$.

Proof. The proof is by induction on $k$. If $k=1$, the statement is true by definition. Let the statement of the theorem be true for $k-1$, and we shall prove it for $k$. If $C \subset \pi_{k-1}^{2}$, the theorem is true, so assume that $C \nsubseteq \pi_{k-1}^{2}$. Let $L_{k}$ be the set of lines which can be adjoined to $\pi_{k-1}^{2}$ in a free extension, and let $\pi_{k-1}(C)=\pi_{k-1}^{2} \cup L_{k} \cup C$. Then $\pi_{k-1}(C)$ is a free extension of $\pi_{k-1}^{2}$, and also of $\pi_{0}^{2}$. By hypothesis, $C$ generates all of $\pi^{2}$, hence, by Theorem 4 , the sub-partial plane of $\pi_{k-1}(C)$ generated by $C$ must be all of $\pi_{k-1}(C)$. For the sake of definiteness let $C$ consist of the points $P_{1}, P_{2}, P_{3}, P_{4}$ The sub-partial plane generated by $C$ can be determined by first adding all lines $P_{i} \cdot P_{j}$ which are in $\pi_{k-1}(C)$, then all points $L_{i} \cap L_{j}$, and so on until the process terminates. If we assume that $k \geqq 2$, then $\pi_{k-1}(C)$ must contain at least eight points (seven in $\pi_{1}^{2}$ and one in $C$ ). But for $C$ to generate points other than $P_{l}$, some of the six lines $L_{i j}=P_{i} \cdot P_{j}=P_{j} \cdot P_{i}$ must be in $\pi_{k-1}(C)$. Also, it is easy to see that at least two of the three diagonal points of $C$ must be in $\pi_{k-1}(C)$ as intersections of the lines $L_{i j}$ if we are to be able to generate more than five points. Observe now that if all three diagional points of $C$ are in $\pi_{k-1}(C)$ as intersections of the $L_{i j}$, all six lines $L_{i j}$ must be in $\pi_{k-1}(C)$. But in this case, every point $P_{i}$ of $C$ will have at least three lines through it which would imply that $C \subset \pi_{k-1}^{2}$, a contradiction. Hence, exactly two diagonal points of $C$ must be in $\pi_{k-1}(C)$ as intersections of the $L_{i j}$. Let us say that these diagonal points are $d_{1}=L_{12} \cap L_{34}, d_{2}=L_{14} \cap L_{23}$. So far, we have six points of $\pi_{k-1}(C)$. To construct another, we must have the line $d_{1} \cdot d_{2}$ intersecting with the line $L_{13}$ or $L_{24}$ (but not both-see Figure 1).

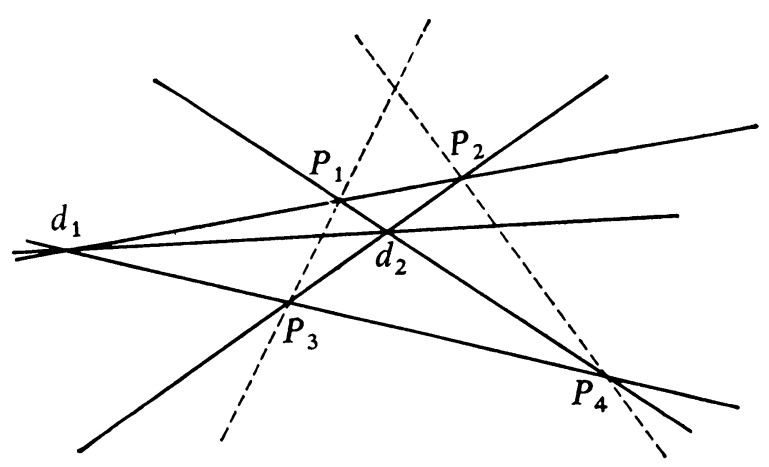

FIGURE 1 
If, for example the line $L_{13}$ is in $\pi_{k-1}(C)$, then the points $P_{1}$ and $P_{3}$ both have three lines through them and are therefore already in $\pi_{k-1}^{2}$. Also, the points $d_{1}$ and $d_{2}$ being in $\pi_{k-1}(C)$ and not in $C$ must be in $\pi_{k-1}^{2}$. Thus the configuration $C^{\prime}=\left\{P_{1}, P_{3}, d_{1}, d_{2}\right\}$ lies in $\pi_{k-1}^{2}$, and generates all of $\pi^{2}$, since it generates $C$ which generates $\pi^{2}$ by hypothesis. Also by the induction hypothesis, there exists a chain of length $k-1$ from $\pi_{0}^{2}$ to $C^{\prime}$, and since all the points of $C$ are contained among the points of $C^{\prime}$ and its diagonal points, the theorem is proved. Observe, incidentally, that the next line created must be either $P_{4} \cdot\left[\left(d_{1} \cdot d_{2}\right) \cap L_{13}\right]$ or $P_{2} \cdot\left[\left(d \cdot d_{2}\right) \cap L_{13}\right]$. Thus either $P_{2}$ or $P_{4}$ has three lines through it, so exactly one point of the four was a newly created point.

We have, in Theorem 6 , given a method for constructing any configuration generating $\pi^{2}$, and hence, any collineation. Our next task is to display explicitly the generators of the collineation group. Since a collineation is determined by its action on the points $A_{i}, B_{j}$, a collineation will be designated by

$$
\left[\begin{array}{lll}
A_{1} & \rightarrow & P_{1} \\
A_{2} & \rightarrow & P_{2} \\
B_{1} & \rightarrow & P_{3} \\
B_{2} & \rightarrow & P_{4}
\end{array}\right]
$$

The group $S^{2}$ is isomorphic with $S_{4}$, and can be generated by the two collineations

$$
\theta_{1}=\left(\begin{array}{l}
A_{1} \rightarrow A_{2} \\
A_{2} \rightarrow A_{1} \\
B_{1} \rightarrow B_{1} \\
B_{2} \rightarrow B_{2}
\end{array}\right), \quad \theta_{2}=\left[\begin{array}{c}
A_{1} \rightarrow A_{2} \\
A_{2} \rightarrow B_{1} \\
B_{1} \rightarrow B_{2} \\
B_{2} \rightarrow A_{1}
\end{array}\right),
$$

where $\theta_{1}^{2}=\theta_{2}^{4}=I$, the identity collineation. Let $a_{1}=A_{1} \cdot A_{2} \cap \mid B_{1} \cdot B_{2}$, $a_{2}=A_{1} \cdot B_{2} \cap A_{2} \cdot B_{1}, a_{3}=A_{1} \cdot B_{1} \cap A_{2} \cdot B_{2}$ be the diagonal points of $\pi_{0}^{2}$. A typical collineation of rank one is

$$
\phi=\left[\begin{array}{l}
A_{1} \rightarrow A_{1} \\
A_{2} \rightarrow a_{1} \\
B_{1} \rightarrow B_{1} \\
B_{2} \rightarrow a_{2}
\end{array}\right]
$$

(Note that $\phi^{2}=I$, for $\left(a_{1}\right) \phi=\left(A_{1}\right) \phi \cdot\left(A_{2}\right) \phi \cap\left(B_{1}\right) \phi \cdot\left(B_{2}\right) \phi=A_{1} \cdot a_{1} \cap B_{1} \cdot a_{2}$ $=A_{2}$, and $\left(a_{2}\right) \phi=\left(A_{1}\right) \phi \cdot\left(B_{2}\right) \phi \cap\left(A_{2}\right) \phi \cdot\left(B_{1}\right) \phi=A_{1} \cdot a_{2} \cap a_{1} \cdot B_{1}=B_{2}$.) We can now prove

THEORFM 7. $G_{2}$ is generated by the collineations $\theta_{1}, \theta_{2}$, and $\phi$.

Proof. First, recall that there are exactly seven configurations of four points in $\pi_{1}^{2}$ which generate $\pi^{2}$. By Theorem 3 , to find all rank 0 or 1 collineations we need only find one coset representative mapping $\pi_{0}^{2}$ onto each of these configurations. For example, 
(5)

$$
\left.\left.\begin{array}{ll}
\theta_{1} \phi \theta_{1}= & {\left[\begin{array}{l}
A_{1} \rightarrow a_{1} \\
A_{2} \rightarrow A_{2} \\
B_{1} \rightarrow B_{1} \\
B_{2} \rightarrow a_{3}
\end{array}\right],} \\
\theta_{2}^{-1} \phi \theta_{2}=\left[\begin{array}{l}
A_{1} \rightarrow a_{1} \\
A_{2} \rightarrow A_{2} \\
B_{1} \rightarrow a_{2} \\
B_{2} \rightarrow B_{2}
\end{array}\right], & \theta_{1} \phi \theta_{1} \theta_{2}^{2}=\left[\begin{array}{l}
A_{1} \rightarrow a_{1} \\
A_{2} \rightarrow B_{2} \\
B_{1} \rightarrow A_{1} \\
B_{2} \rightarrow a_{3}
\end{array}\right], \\
\theta_{1} \phi \theta_{1} \theta_{2}=\left[\begin{array}{l}
A_{1} \rightarrow A_{1} \\
A_{2} \rightarrow a_{2} \\
B_{1} \rightarrow B_{2} \\
A_{2} \rightarrow B_{1} \\
B_{1} \rightarrow B_{2} \\
B_{2} \rightarrow a_{3}
\end{array}\right], & \theta_{2}^{-2} \phi \theta_{2}^{2}=
\end{array}\right], \begin{array}{l}
A_{1} \rightarrow a_{2} \\
A_{2} \rightarrow A_{1} \\
B_{1} \rightarrow A_{2} \\
B_{2} \rightarrow a_{3}
\end{array}\right] .
$$

These six collineations, together with the identity, give a set of coset representatives for the seven cosets which the rank 0 and 1 collineations constitute. The remainder of the proof proceeds by induction on the length of a chain between $\pi_{0}^{2}$ and the configuration giving rise to the collineation which we are trying to write in terms of $\theta_{1}, \theta_{2}$, and $\varphi$. Let $C$ be the configuration into which this collineation, $\alpha$, maps $\pi_{0}^{2}$ :

$$
\alpha: \pi_{0}^{2} \rightarrow C \text {. }
$$

Then, since $C \subset \pi_{0}^{n}$ for some $n$, by Theorem 6 there exists a configuration $C^{\prime} \subset \pi_{0}^{n-1}$ such that the points of $C$ are contained among the points and diagonal points of $C^{\prime}$. By the induction hypothesis, then, there is a collineation, $\beta$, generated by $\theta_{1}, \theta_{2}$, and $\phi$ with

$$
\beta: \pi_{0}^{2} \rightarrow C^{\prime}
$$

Now, the collineation $\gamma=\beta^{-1} \alpha$ maps $C^{\prime}$ onto $C$ :

$$
\gamma: C^{\prime} \rightarrow C \text {. }
$$

But $\beta \gamma \beta^{-1}$, being a conjugate of $\gamma$ (which maps $C^{\prime}$ into itself and its diagonal points), must map $\pi_{0}^{2}$ into $\pi_{1}^{2}$, and hence, $\beta \gamma \beta^{-1}=\eta$, which is in one of the seven cosets of $S^{2}$ given by the identity and (5). Thus,

$$
\beta^{-1} \eta \beta=\beta^{-1} \alpha,
$$

where $\beta$ and $\eta$ are generated by $\phi, \theta_{1}$, and $\theta_{2}$, which completes the proof of the theorem.

Indeed, it follows from (9), that $\alpha$ is a product of no more than $n$ rank 0 or 1 collineations if $\alpha$ maps $\pi_{0}^{2}$ onto a configuration at the other end of a chain of length $n$.

Another question which can be asked about the collineation group $G_{2}$ concerns the orbits of the points of $\pi^{2}$ under $G_{2}$. Clearly the orbit containing the four 
original generators of $\pi^{2}$ consists of exactly those points which lie on some generating quadrilateral. The following theorem characterizes this set of points.

THEOREM 8. Let $P$ be a point of $\pi^{2}$ which is in $\pi_{k}^{2}$, but not in $\pi_{k-1}^{2}$. Thus $P=L_{1} \cap L_{2}, L_{i} \in \pi_{k}^{2}$. Then $P$ lies on a generating quadrilateral if and only if there is a generating quadrilateral $\pi_{k-1}^{2}$, two points of which are incident with $L_{1}$, and two with $L_{2}$.

Proof. The first ("if") part of the theorem is obvious. To prove the converse, assume that $P$ does lie on a generating quadrilateral $C \subset \pi_{n}^{2}$, and that $P$ lies on no such generating quadrilateral of $\pi_{n-1}^{2}$. Clearly $n \geqq k$. We refer to Figure 1 and let $P=P_{4}$ where $C=\left\{P_{1}, P_{2}, P_{3}, P_{4}\right\}$. By the remarks following the proof of Theorem 5 , exactly one of the $P_{i}$ does not already belong to $\pi_{n-1}^{2}$.

Case 1. $P \in \pi_{n-1}^{2}$. By Theorem 6 there exists a generating configuration $C^{\prime} \subset \pi_{n-1}^{2}$ consisting of two of the three $P_{i}$ which are in $\pi_{n-1}^{2}$ and two of the diagonal points of $C$. If $P=P_{4}$ is one of the points of $C^{\prime}$, our choice of the configuration $C$ as the generating quadrilateral containing $P$ and of "least degree" is violated. Thus, we must have the situation in Figure 1, where $C^{\prime}=\left\{d_{1}, d_{2}, P_{1}, P_{3}\right\}$ and $P_{2}$ is the point $\notin \pi_{n-1}^{2}$. But for $C^{\prime}$ to be a generating quadrilateral, the point $\left(d_{1} \cdot d_{2}\right) \cap L_{13}=Q$ must be in $\pi_{n-1}^{2}$ (see the Proof of Theorem 6) which means that the generating configuration $C^{\prime \prime}=\left\{Q, P, d_{2}, P_{3}\right\}$ is contained in $\pi_{n-1}^{2}$, again contradicting our choice of $C$.

Case 2. $P \notin \pi_{n-1}^{2}$. Thus, $k=n$, and by Theorem 6, there exists a generating quadrilateral contained in $\pi_{n-1}^{2}$ with $P$ as one of its diagonal points. But clearly the only way $P$ can be the diagonal point of a quadrilateral of $\pi_{n-1}^{2}$ is if this quadrilateral generates in $\pi_{n}^{2}$ the two lines which have created $P$ and the proof is thus complete.

To see that there are points which lie on no generating quadrilateral, it suffices to choose four points of some $\pi_{k}^{2}$ no two of which lie on the same line of $\pi_{k}^{2}$. Then none of the three diagonal points of such a quadrilateral can lie on a generating quadrilateral. Four such points can be chosen when $k \geqq 2$.

4. $n>2$. In this case, the group of collineations of $\pi^{n}, H_{n}$, generated by the rank 0 and rank 1 collineations, is somewhat more difficult to describe, and the following notation will be introduced to discuss the points and lines of $\pi_{1}^{n}$.

lines:

$$
\begin{aligned}
& L_{1}=A_{1} \cdots A_{n}, \\
& L_{2}=B_{1} \cdot B_{2}, \\
& L_{i j}=A_{i} \cdot B_{j}, i=1,2, \cdots, n ; j=1,2 .
\end{aligned}
$$

points:

$$
\begin{aligned}
A_{i}, i & =1,2, \cdots, n, \\
B_{j}, j & =1,2, \\
A_{n+1} & =L_{1} \cap L_{2}, \\
P_{i j k l} & =P_{k l l j}=L_{i j} \cap L_{k l} .
\end{aligned}
$$


Observe that there are $n+1$ points on $L_{1}$, three points on $L_{2}$, and $n+1$ points on $L_{i j}$. If $i=k, P_{i j k l}=A_{i}$, and if $j=l, P_{i j k l}=B_{j}$. Furthermore, $P_{i j k l}$ and $P_{a b c d}$ are on a line of $\pi_{1}^{n}$ if and only of $(i, j)=(a, b)$ or $(c, d)$, or $(k, l)=(a, b)$ or $(c, d)$. With this notation we can describe those configurations in $\pi_{1}^{n}$ which generate $\pi^{n}$. For the moment, since the line $L_{2}$ introduces extra configurations when $n=3$, we shall assume $n>3$.

The lines which are candidates for being images of $L_{1}$ are $L_{1}$ and the $L_{i j}$. If $L_{1}$ is chosen, the set of $n$ points can either be $A_{1}, \cdots, A_{n}$, or it can include $A_{n+1}$. In the former case, the two points can be $B_{1}, B_{2}$ (yielding $\pi_{0}^{n}$ ), or any two points $P_{i j k l}, P_{a b c d}$ not on a line of $\pi_{1}^{n}$. To see this, observe that if $P_{i j k l}, P_{a b c d}$ are not on a line of $\pi_{1}^{n}$, neither can be a point $B_{i}$, so $j \neq l, b \neq d$. Let $j=b=1, l=d=2$. Then $B_{1}=L_{i 1} \cap L_{a 1}, B_{2}=L_{k 2} \cap L_{c 2}$. On the other hand, if two points ( $\neq B_{1}, B_{2}$. are on a line of $\pi_{1}^{n}$, they must be on some $L_{i j}$, and hence collinear with some $A_{i}$ and the whole configuration could not possibly generate a $\pi^{n}$. By a similar argument, it can be seen that if $A_{1}, \cdots, A_{n-1}, A_{n+1}$ are taken to be the $n$ points, the two points can either be two points collinear with $A_{n}$, or $P_{i 1 k 2}, P_{a 1 c 2}$ not on a line of $\pi_{1}^{n}$, as long as (a) neither $i=c=n$ nor $a=k=n$ is satisfied; (b) one of $i, c, a, k$ is equal to $n$. For if neither $i=c=n$ nor $a=k=n$ holds, then either the pairs of lines $A_{a} \cdot P_{a 1 c 2}$ and $A_{i} \cdot P_{i 1 k 2}$ or the lines $A_{c} \cdot P_{a 1 c 2}$ and $A_{k} \cdot P_{i 1 k 2}$ can be defined and their intersection will yield $B_{1}$ or $B_{2}$, say $B_{1}=A_{a} \cdot P_{a 1 c 2} \cap A_{i} \cdot P_{i 1 k 2}$. But now $L_{2}=B_{1} \cdot A_{n+1}$, and $B_{2}=L_{2} \cap\left(A_{c} \cdot P_{a 1 c 2}\right)$ or $L_{2} \cap\left(A_{k} \cdot P_{i 1 k 2}\right)$, and finally if $i, c, a$, or $k$ is $n$, a line through $B_{1}$ or $B_{2}$ and $P_{a 1 c 2}$ or $P_{i 1 k 2}$ will meet $L_{1}$ in $A_{n}$. If, on the other hand, $i=c=n$, only the lines $A_{a} \cdot P_{a 1 c 2}$ and $A_{k} \cdot P_{i 1 k 2}$ can be drawn from the set of $n+2$ points, and only their point of intersection $P_{a 1 k 2}$ will be added, proving that the configuration will not generate all of $\pi^{n}$. Finally, if $i, c, a$, and $k$ are all distinct from $n$, the point $A_{n}$ can never be generated by the given configuration

If some $L_{i j}$, say $L_{11}$, is the line which is the image of $L_{1}$ under the collineation, there are three possibilities for the set of $n$ points:

(1) the set of points includes both $A_{1}$ and $B_{1}$;

(2) only $A_{1}$ is included;

(3) only $B_{1}$ is included.

We shall cover these cases separately and decide which points can be used as the two points in the various configurations. Under case (1), we can choose the points $A_{1}, B_{1}, P_{11 i 2}, i \neq 1, n$, as the $n$ points. For the other two points, we can either choose any two points $\left(\neq P_{11 n 2}\right)$ on the line $L_{n 2}$, so long as one of them is $B_{2}$ or $A_{n}$; we can have any two points $P_{i 1 k 2}, P_{a 1 c 2}$ not on a line of $\pi_{1}^{n}$ so long as (a) $k, c \neq n$, and (b) $i=n$ or $a=n$; or we can have the point $A_{n+1}$ together with a point on $L_{n 2}$. To see this when the two points are on $L_{n 2}$ let $P_{a 1 n 2}, P_{b 1 n 2}$ be any two points $\left(\neq P_{11 n 2}\right)$ on $L_{n 2}$, and assume that $a, b \neq n$. Then the lines $L_{n 2}, L_{a 1}, L_{b 1}$ and the point $P_{11 n 2}$ are generated, but the process goes no further. If $a=n$, how- 
ever, the lines $L_{n 2}, L_{1}=A_{1} \cdot A_{n}, L_{b 1}=B_{1} \cdot P_{b 1 n 2}$, and the point $A_{b}=L_{b 1} \cap L_{1}$ are generated. Then, $B_{2}=L_{n 2} \cap\left(A_{b} \cdot P_{11 b 2}\right)$, and all of $\pi_{1}^{n}$ is easily generated. Similarly, when one of the points is $B_{2}, \pi_{1}^{n}$ is generated readily. If, on the other hand, the two points are taken to be $P_{i 1 k 2}, P_{a 1 c 2}, i \neq a, k \neq c$, then if $c, k \neq n$, $L_{k 2}=P_{11 k 2} \cdot P_{i 1 k 2}, L_{c 2}=P_{11 c 2} \cdot P_{a 1 c 2}$, and $B_{2}=L_{k 2} \cap L_{c 2}$. Then if $i=n$ or $a=n$, this case reduces to two points on $L_{n 2}$ as above. If neither $i=n$ nor $a=n$, the point $A_{n}$ will never be generated, and the configuration cannot generate all of $\pi_{1}^{n}$. Furthermore, if, say, $k=n$, the only lines generated would be $L_{i 1}=B_{1} \cdot P_{i 1 k 2}$, $L_{a 1}=B_{1} \cdot P_{a 1 c 2}, L_{c 2}=P_{11 c 2} \cdot P_{a 1 c 2}$, and the only points generated would be $P_{i 1 c 2}=L_{i 1} \cdot L_{c 2}$, thus again all of $\pi_{1}^{n}$ would not be generated. Finally, if $A_{n+1}$ is one of the points, the lines $L_{1}=A_{1} \cdot A_{n+1}$ and $L_{2}=B_{1} \cdot A_{n+1}$ are generated. Also, if $P_{i 1 n 2}$ is the point on $L_{n 2}, A_{i}=L_{1} \cap\left(B_{1} \cdot P_{i 1 n 2}\right)$, and $B_{2}=L_{2} \cap\left(A_{i} \cdot P_{11 i 2}\right)$, and we are in the same situation as previously.

In case (2), let the $n$ points be $A_{1}, P_{11 i 2}$. As the two points, we can have any two points $(\neq B)$ on a line $\left(\neq L_{11}\right)$ through $B_{1}$, or some point $A_{i}\left(\neq A_{n+1}\right)$ and any point $P_{a 1 k 2}(k \neq i)$. Finally, in case (3), if the $n$ points are $B_{1}, P_{11 i 2}$, the two points can be any two points $\left(\neq A_{1}\right)$ on $L_{1}$; or $B_{2}$ and any point $\left(\neq A_{1}\right)$ on $L_{12}$; or two points $P_{i 1 k 2}, P_{a 1 c 2}$ not on a line of $\pi_{1}^{n}$, such that $i=c$ and $a=k$ and such that $k, c \neq 1$; or $A_{n+1}$ and any point not on $L_{11}$ or $L_{12}$. The proofs in these cases are essentially the same and require a case analysis similar to the ones carried out above.

When $n=3$, a further line, $L_{2}$, can be an image of $L_{1}$, and the other two points can be any two points $P_{i 1 k 2}, P_{a 1 c 2}$ not on a line of $\pi_{1}^{3}$, as long as the equalities $i=c, k=a$ are not both satisfied.

The cases considered above allow one to explicitly write out a set of generators of $H_{n}$. They will not be listed here, but the list consists of three generators for $S_{n} \times S_{2}$, and representatives of the different types of collineations. We shall prove, however,

THEOREM 9. There exists an integer $m$ independent of $n$ such that $H_{n}$ is generated by $m$ collineations for any $n \geqq 3$.

Proof. Assume $n>3$, for $H_{3}$ is certainly finitely generated. If $A_{1}, \cdots, A_{n}$ are the $n$ points, then the two points (if they are not $B_{1}, B_{2}$ ) are of the form $P_{i 1 k 2}$, $P_{a 1 c 2}, i \neq a, k \neq c$, and are of three types:

(a) $1 \neq c, k \neq a$;

(b) $i=c, k \neq a$, or $i \neq c, k=a$;

(c) $i=c, k=a$.

Now, three collineations all fixing the $A_{i}$ and one mapping $B_{1}, B_{2}$ into each of these types of pairs of points, $P, P^{\prime}$ will generate all the collineations of rank 1 having the set $\left\{A_{i}\right\}$ as the $n$ points. To see this, for example, assume we wish to generate the collineation $A_{i} \rightarrow A_{i}, B_{1} \rightarrow P_{x 1 y 2}, B_{2} \rightarrow P_{z 1 w 2}, x \neq w, y \neq z$. To do 
this, conjugate the given collineation $\phi: A_{j} \rightarrow A_{j}$, all $j, B_{1} \rightarrow P_{i 1 k 2}, B_{2} \rightarrow P_{a 1 c 2}$ by the collineation $A_{i} \rightarrow A_{x}, A_{k} \rightarrow A_{s}, A_{a} \rightarrow A_{z}, A_{c} \rightarrow A_{w}$, in $S^{n}$. Pre-multiplication by elements of $S^{n}$ will give all possible ways of mapping $\left\{A_{i}\right\}$ into $\left\{A_{i}\right\}$, and both ways of mapping $B_{j}$ into $\left\{P_{x 1 y 2}, P_{z 1 w 2}\right\}$. A similar argument holds for each of the cases (1), (2), (3) above. For example, if a collineation $\phi$ is given which maps $L_{1}$ onto $L_{11}$, a collineation $\phi^{\prime}$ mapping $L_{1}$ into $L_{i j}$ can be obtained by conjugating $\phi$ by the collineation $A_{1} \rightarrow A_{i}, B_{1} \rightarrow B_{j}$. Thus, every collineation of rank 1 can be seen to be generated from a fixed number of collineations of rank 1 , no matter which free plane is under consideration.

5. Comments. In $\S 3$, we have obtained generators for the collineation group of $\pi^{2}$. In $\S 4$, it was shown that the group of collineations of $\pi^{n}, n>2$, generated by the rank 0 and rank 1 collineations always has less than a fixed number of generators. The generators were not explicitly written down, as it was felt that the space they would consume would not be justified inasmuch as $\S \S 2,3$, and 4 make evident the methods for obtaining them quite easily. A further omission was the relations holding between the generators of $G_{2}$. A finite set of relations (possibly not minimal) is known to the author, but the proof that they suffice to determine the group is too long and complicated for inclusion here. Finally, the large question left unanswered by this paper is the equality of $H_{n}$ and $G_{n}$ for $n>2$. The author's conjecture is that they are equal, but the technique of Theorem 6 cannot be used in a proof for the general case.

\section{REFERENCES}

1. Marshall Hall, Jr., Projective planes, Trans. Amer. Math. Soc. 54 (1943), 229-277.

2. D. R. Hughes, On homomorphisms of projective planes, Proc. Sympos. Appl. Math., Vol. 10, pp. 45-52, Amer. Math. Soc., Providence, R. I., 1960.

INSTITUTE FOR DEFENSE ANALYSES, Princeton, New Jersey 